\title{
Haptic Feedback and Human Performance in a Dynamic Task
}

\author{
Felix Huang R. Brent Gillespie Art Kuo \\ Department of Mechanical Engineering, University of Michigan, Ann Arbor, MI 48109 \\ fhuang@umich.edu; brentg@umich.edu; artkuo@umich.edu
}

\begin{abstract}
This study explores the effects of haptic feedback on performance and learning by human subjects executing a dynamic task. We present the results of experiments involving the control of a ball and beam. Human subjects perform position targeting of the ball through hand operation of the beam angle. In our dynamic analysis we discuss how this prototype task may be used to test the efficacy of various haptic feedback conditions. We obtain results for two conditions of haptic feedback, produced using two ball sizes, and apply various metrics to analyze performance. We also examine ordering effects that occur in the presentation of these haptic conditions. Our analysis and experimental findings indicate that the performance of a dynamic task is governed by the complexity of system dynamics and the magnitude of haptic feedback. Our results provide modest support to recommend exposure to a more complex, higher force-feedback task prior to the execution of a simpler lower feedback task.
\end{abstract}

\section{Introduction}

The addition of force feedback has been shown to increase the efficacy of certain virtual environments for training cognitive (Brooks, 1990) and manual (Hasser, 1998) skill. Certainly one's sense of presence and immersion is enhanced when force feedback is included, since one's basis for comparison is experience in the physical world, where reaction forces are generally available. Experience within the prosthetics community has also shown that force feedback is critical for promoting impressions that the object in one's grasp is an extension of one's own body (Childress, 1986). Likewise, the success of skill transfer from a virtual to a physical environment might be expected to rely on the availability of haptic feedback in the virtual environment. Adams, Klowden and Hannaford explored the efficacy of force feedback in the training of assembly tasks. This study involved a complex manipulation task that engaged multiple manual skills. Their results were inconclusive as to whether force feedback resulted in performance gains (Adams, 2001). Repperger examined the performance of humans in a discrete aiming task in terms of information processing rate (Repperger, 1995). More accurate aiming was achieved under the influence of non-linear spring forces from a haptic interface. Mussa-Ivaldi showed that the application of 'force fields', through a haptic interface, caused humans to adapt to the novel conditions and perform desired motions given sufficient practice. Such a training regimen was shown to have some lasting effects even after the field was removed, suggesting that humans were making use of internal models of the force fields developed during training (Mussa-Ivaldi, 2000). The above experiments, however, describe tasks in which the haptic feedback was a simple function of the human's hand position or velocity.

Human operation of dynamic systems, however, requires more complex interaction than in static ones. Schaal studied human performance in a dynamic task involving rhythmic juggling with a ball and paddle. He showed that in the chosen dynamical task, humans exploit stable solutions inherent to the system by selecting a negative impact acceleration between ball and paddle (Schaal, 1996). Yamshita et. al. studied learning of manual control of a crane mechanism. His analysis suggested that the solutions produced by human operators were heuristically developed and eventually equivalent to certain optimal control solutions (Yamashita. 1983). His results also demonstrated that the degree of difficulty of a task affects the ability of the human in acquiring proficiency.

These studies of dynamic systems show the importance of the system characteristics for manual control. In the current study, we will address what characteristics set dynamic systems apart from static systems, and what factors in a dynamic system contribute to task difficulty. In particular, we are interested in quantifying the value of force feedback in manual control of dynamical systems. We investigate how performance in a dynamic manual task is affected by various task conditions, including the magnitude of force feedback and task complexity.

Unlike static systems, a dynamic system effectively has memory: its current state is not only a function of current input, but also past input. 
Therefore, a human operator wishing to drive a dynamic system to a desired state ideally should command input to the system in a way that respects its history and its dynamical response, rather than in an ad hoc, spontaneous way. Thus he stands to achieve a more optimal control of the dynamic system in terms of applied effort or efficiency, to reaching the desired system state.

Although for many common tasks the primary mode of sensing may be visual, the haptic mode may be important in the control of dynamic systems. Humans possess the ability, through haptic interaction alone, to identify mechanical properties of physical objects. In principle, mechanical properties of a system such as the inertia, damping, and stiffness may be ascertained visually, by observing how an object responds to a known force over time. These properties, however, are more readily identified when the human probes mechanically with a finger, sensing both force and motion in a collocated fashion.

Consider a simple dynamic system: a block of mass $M$ sliding without friction with velocity $V$ under the action of a force $F$ applied by a human, as shown in Figure 1:

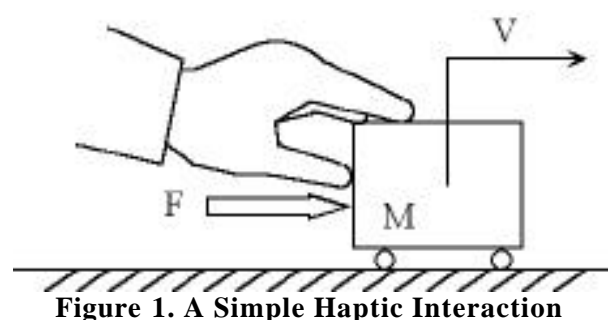

$$
V(s)=\frac{1}{M s} F(s)
$$

A human operator must apply a force trajectory that, if integrated, will produce the desired velocity trajectory. To accomplish this action, he must either plan ahead using recall of past information, or somehow use a real-time process akin to differentiation of the desired velocity (assuming open loop control) to accomplish his goal. Through interaction with the block, the human operator may obtain useful information about its characteristics, in particular its mass. Such information, along with previous experience, enables planning and precludes the need for closed loop control of the system involving real-time differentiation of sensed velocity.

In this example, the use of a larger mass may actually produce deteriorated performance for the control of motion, because of the greater required effort. But the utility of a larger mass may lie in its ability to present a greater challenge to the human and thereby create an opportunity for accelerated learning. Additionally, experience with similar dynamical systems (inertias or other integrators), along with force feedback might provide assistance for planning appropriate input force time-histories for a novel task. Therefore, for dynamic systems in general, we must ask what type of haptic conditions provides the optimal environment for not just achieving performance goals, but for improving human skill. Equation (1) illustrates a relationship between the human and the manipulandum that, though simple, still requires identification of the system characteristics, i.e. the mass, for proper control. For more complex dynamic systems, the human would be even more reliant on knowledge of the system.

Certainly haptic feedback can be considered another information channel to augment vision or sound. But given the inevitable pairing of force and motion at the "driving point" where information transmission occurs, haptic information is always accompanied by power transfer.

Inevitably, a mechanical contact also supports dynamical coupling between the communicating systems. The coupled dynamical system formed by the introduction of mechanical communication can in fact display altogether different behaviors than the two systems are capable of displaying separately. These two distinct modes of communication, information and power transfer, may have implications that are variously positive or negative when taken in the context of a particular task. Quite possibly the information is useful, but the power transfer only serves to drive the coupled system away from the task goal. More simply, increased force feedback may require an increased amount of work to accomplish the same goal.

Another important axis on which to consider the role of force feedback is in motor control programs that may be first developed using closed loop control but then proceed to open loop, or automatic control. The value of force feedback may diminish as motor learning takes place. The existence of a deleterious effect of force feedback such as undesirable dynamical coupling or increased manual workload then constitutes a tradeoff that must be balanced.

To explore the various roles of force feedback and to begin to assign quantitative value to them in the context of a dynamical task, we have developed a computer-controlled ball and beam device, in which a subject balances a ball on a rotating see-saw. This task can be performed on the actual device, or in a virtual environment where the subject sees a ball and beam on a computer screen, but feels suppressed or enhanced force feedback. 
We have chosen as our focus of study a system that displays continuous second-order system dynamics and that does not feature changing contact conditions. We define a quantitative performance measure together with a task involving manual control of the beam. We suspect that force feedback plays an important role in learning to stabilize the ball and beam system, and in this paper we present an experiment designed to either confirm or deny that suspicion. We also explore the effects of ordering, that is, of presenting one of two forcefeedback conditions prior to the other.

In the following sections, we present the mechanics of the ball and beam, including an expression for force-feedback as a function of state variables and system parameters, we present our experimental methods and results, and finally we discuss our findings before briefly outlining future work.

\section{Theoretical Development}

In the following section we provide an analysis of how the dynamics of our prototype task allows for an appropriate test of the influence of haptic feedback. We develop the equations of motion that govern the dynamics of the ball, and the equations of haptic interaction that describe the feedback to the human operator.

\subsection{Ball and Beam Equations of Motion}

Consider the dynamic system depicted in Figure 2 below. A ball of radius $R$ rolls without slipping on a beam, whose angular displacement relative to the ground is $\grave{e}$. The beam is configured such that when the ball position $r=0$, the ball center is coincident with the beam pivot. The angular displacement $\hat{a}$ of the ball relative to the beam is related to the displacement $r$ of the ball center to the beam pivot by the rolling constraint:

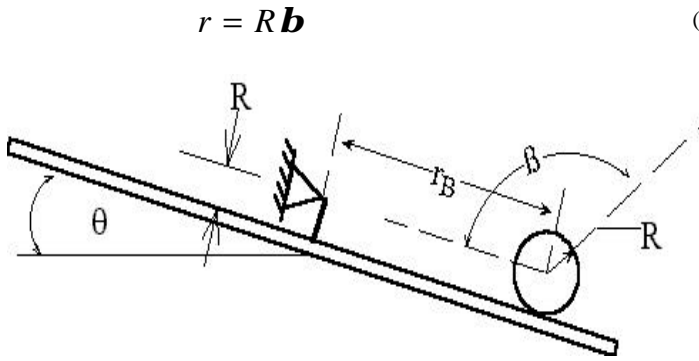

Figure 2. Schematic of Ball and Beam System

For this analysis, we will consider the human operator to be a flow source, capable of producing any desired time history $\grave{e}(t)$ and its time derivatives. The rolling constraint (Eq. 2) can be used to eliminate the dependent generalized coordinate $\beta$, leaving the single independent generalized coordinate $r$. A rolling object has a mass moment of inertia

$$
I=m k^{2}=\alpha m R^{2},
$$

where $m$ is the mass of the ball or other rolling object, $k$ is the radius of gyration, and á is a dimensionless factor ( $2 / 5$ for a sphere) dependent on geometry. The kinetic energy $\mathrm{T}$ and potential energy $\mathrm{V}$ of the system are

$$
\begin{gathered}
V=-m g r \sin \theta(t) \\
T=\frac{1}{2} m\left[\left(r^{2}+\alpha R^{2}\right) \dot{\theta}(t)^{2}+2 \alpha \operatorname{R} \dot{r} \dot{\theta}(t)+(1+\alpha) \dot{r}^{2}\right]
\end{gathered}
$$

Using Lagrangian methods, the following equation of motion is formed:

$$
\begin{array}{r}
r=\lambda\left(g \sin \theta+r \dot{\theta}^{2}-R \ddot{\theta}\right) \\
\lambda=(1+\alpha)^{-1}=\frac{5}{7} \text { for a sphere. }
\end{array}
$$

It is apparent from the above relations that any two balls with the same outer radius $R$ and radius of gyration $k$ will behave alike, regardless of their mass.

As can be seen in the equation of motion, the ball radius $R$ scales the contribution of the beam angular acceleration $\ddot{\boldsymbol{\theta}}$ to ball acceleration. For small ball radii, motion is dominated by the gravity term. However for larger ball radii, more complex dynamics are manifested as the ball acceleration $\ddot{r}$ becomes dependent on the beam angular acceleration $\ddot{\boldsymbol{\theta}}$. After linearization of the equation of motion, we see that as the ball radius $R$ approaches zero, the acceleration $\ddot{r}$ becomes proportional to the angle $\Theta(t)$ :

$$
\ddot{r} \approx(\lambda g) \theta(t)
$$

The above equation shows that a small ball behaves like a free falling object in a gravity field modulated by $\Theta(t)$. Thus, this portion of the equation of motion describes the gross motion of the ball across the beam. As the ball increases in size, however, the ball acceleration $\ddot{r}$ becomes increasingly coupled to the beam acceleration $\ddot{\boldsymbol{\theta}}(t)$ as shown in Equation 6 .

As the ball radius $R$ further increases, the equation reduces to a simple kinematic system $\ddot{r}=\lambda R \ddot{\theta}(t)$. 
It is the contribution of both terms that occur for intermediate ball diameters that will increase the complexity of haptic interaction and prove a greater challenge to the human operator.

\subsection{Equations of Haptic Interaction}

For the human operator, the ability to control the ball dynamics may depend on his ability to haptically sense the state of the system. Using Lagrangian methods, and expanding on the analysis of our dynamic system with the use of an undetermined multiplier, we develop the following equation describing the torque that the human feels in response to the angle inputs applied:

$\tau(t)=I_{B} \ddot{\theta}(t)+m\left[\alpha R^{2}\left(\ddot{\theta}(t)+\frac{\ddot{r}}{R}\right)+r^{2} \ddot{\theta}(t)+2 r \dot{r} \dot{\theta}(t)-g r \cos \theta(t)\right]$

The mass of the ball acts as a multiplier on all terms except on the first, the inertia torque of the beam. Thus, the information about the state of the system, including the ball position $r$ and its time derivatives is available to the human through the haptic feedback of the beam torque. Note that the inertia of the beam effectively can mask the torque produced from the ball motion if the mass of the ball is too low.

\subsection{Summary of Haptic Conditions}

The choice of ball mass and choice of ball size both change the conditions of haptic feedback that the human will experience. We associate ball mass with level of force feedback (see Eq 8) and associate ball size with the level of dynamical complexity (see Eq. 6, 7) and lay out these two influences in a table as shown in Table 1.

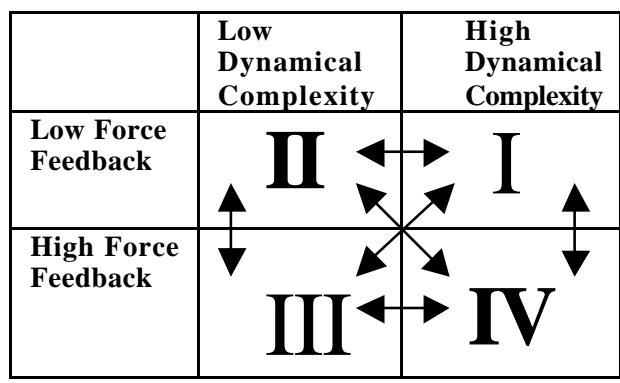

Table 1. Matrix of Haptic Conditions and Testing Sequences

Table 1 presents a two-by-two experiment design to test the influences of force feedback and dynamical complexity. We have labeled the quadrants I, II, II, and IV as shown. For the ball and beam system, mass scales the force feedback, as described in Equation 8, and thus determines the rows of Table 1, either of high or low force feedback. On the other hand, the ball radius $R$ scales the contribution of $\ddot{\theta}(t)$ to $\ddot{r}$, relative to the contributions of the other terms in Equation 6, and thus determines columns of the table, either of low or high dynamical complexity.

We see that the extreme cases are for low force feedback and dynamical complexity (II) and high force feedback and dynamical complexity (IV). We can realize these haptic conditions with the simple choice of two uniform, solid spheres of different diameters. A small diameter ball should correspond to the case of low magnitude of force feedback and should approximate the dynamic behavior of Equation 7. A larger diameter ball should correspond to the case of high magnitude of force feedback and should behave according to the dynamic behavior of Equation 6.

Also in Table 1, we indicate with arrows the possible sequences of any two combinations of haptic condition. Each sequence, along with its reverse direction, could be used to design an experiment to demonstrate which haptic condition produces better performance. In the experiment presented in this paper, we are testing the 'II' and 'IV' conditions in both orders.

\section{Experimental Methods}

\subsection{Description of Apparatus}

Our experimental apparatus consists of an aluminum L-channel beam, 0.8 meter in length, pivoted about its center to a cable drive system and support tower. A motor, not used in this experiment, is connected to the cable drive system. The motor is envisioned for the use in future experiments involving virtual balls, presented with or without force feedback and governed by fully programmable laws of physics. The figure below shows the apparatus and typical operation.

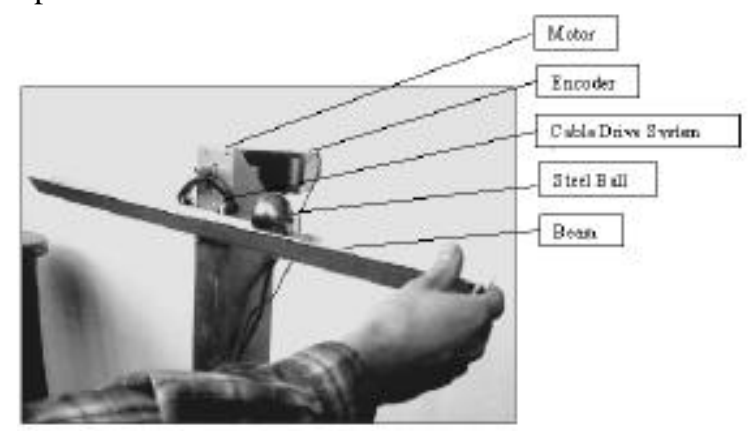

Figure 3. Ball and Beam Apparatus 
For the rolling ball, we used two chrome-steel ball bearings, masses $230 \mathrm{~g}$ and 15 grams, with radii $15.9 \mathrm{~mm}$ and $6.3 \mathrm{~mm}$, respectively. An encoder on the motor shaft measures the angle of the beam. To measure the position of the ball, a linear potentiometer is utilized. A powered conductive strip on an inside edge of the beam serves as the resistive element; the opposite edge of the beam serves as the output lead; and the rolling ball serves as the wiper. The sampling rate of the sensors is 1 $\mathrm{kHz}$.

\subsection{Experimental Protocol}

This experiment consisted of two sessions for each participant, 15 step response trials of Condition-II, and 15 trials of Condition-IV, corresponding to the small and large ball sizes. The sequence of haptic conditions was selected randomly for each participant, and 10 subjects participated in the study. Two subject groups were formed corresponding to the session order, the 'IVII group' and the 'II-IV group'. The randomization of the haptic condition sequence was used to address the effects of ordering so that a fair comparison can be made between the merits of the II and IV conditions.

Each participant was given the same instructions for body stance and hand positioning on the beam and the criteria for performance was explained. There was no prompting of strategy to the participants, and no demonstration of the control action was performed. In the experiment task, the goal was to control the motion of a beam so that a rolling ball moves from a resting position at the center of the beam to a target position located $20 \mathrm{~cm}$ to the right of center along the beam, and then remains at rest. Each subject was informed that performance would be judged primarily on accuracy, and secondarily on speed of response, where 'Accuracy' is defined as the ball proximity to the target position. 'Speed' is defined as the time required to achieve good accuracy. Each trial lasted approximately 30 seconds. There was no rest period between trials, nor between each session testing a certain condition. The ball position and beam angle histories were recorded, and error analyses were conducted as described in section 4 .

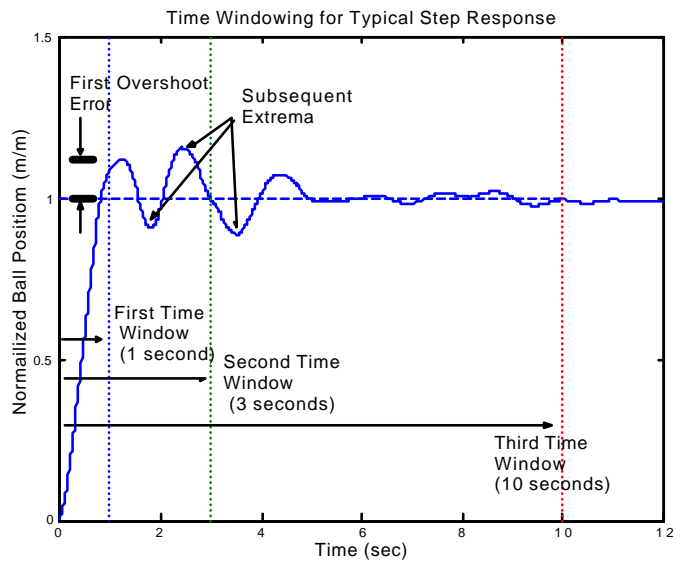

Figure 4. Human Control Typical Ball Response: plot of position as a Function of Time

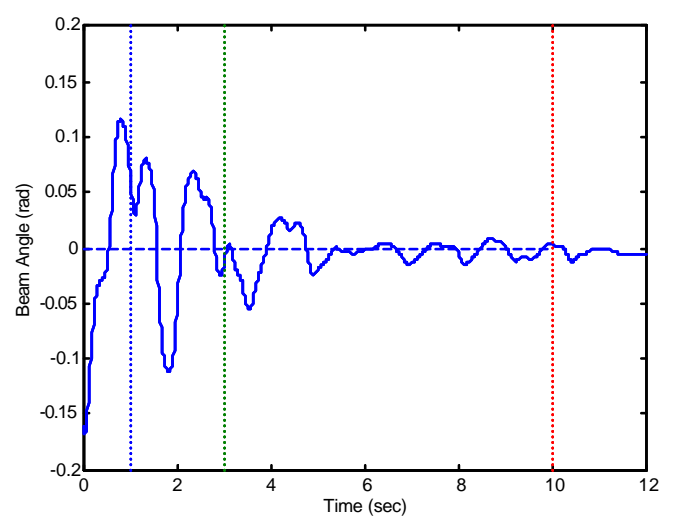

Figure 5. Human Control Typical Ball Response: plot of beam angle as a Function of Time

\subsection{Results}

\subsection{Development of Performance Metrics}

In Figure 4, we present a typical plot of ball position $r$ and beam angle è under human operation for a single trial. Note that there are multiple oscillations in $\mathrm{r}$, and that the oscillations are not exponentially decaying. Also note that è continues to move with appreciable magnitude, even after the ball begins to settle around its goal. Because a typical human control action does not conform well to characteristics of a linear controller, especially for the case of an operator in a novel task, we employ more general methods of error assessment as measures of performance. We apply metrics based on the normalized RMS error of the ball position, as well as the overshoot error (defined below), throughout various time intervals of the position response. For each step response, the RMS error is calculated as: 


$$
e r r_{r m s}=\sqrt{\frac{1}{k_{w}} \sum_{k=1}^{k_{w}}\left(R_{r e f}-y_{k}\right)^{2}}
$$

where $k$ is a time step, and $k_{w}$ is the limit of the chosen window of analysis. Each time step corresponds to a measurement at the system sampling frequency. We can now form performance measures for any time window of interest. The choice of a small window of time at the outset of control is a measure of the speed of response. A larger window of time, though still affected by the speed of response, will have greater weighting towards the settling behavior of the response. We chose three time windows of $\mathrm{t}_{\mathrm{w}}=1,3$, and 10 seconds, which roughly correspond, respectively to rise, intermediate, and settling behavior. To further analyze differences in oscillatory responses, we examine the overshoot error, calculated as absolute difference between the first peak of oscillation and the target position.

\subsection{Analysis of Experimental Data}

We present a comparison of performance results of Condition-IV and Condition-II from averaged data combined from both subject groups. The results are shown for the first three-second time window of RMS error in Figure 5 and for the first overshoot error in Figure 6. The trends found for these metrics were consistent over the other intervals of analysis $\left(\mathrm{t}_{\mathrm{w}}=1,10\right)$.

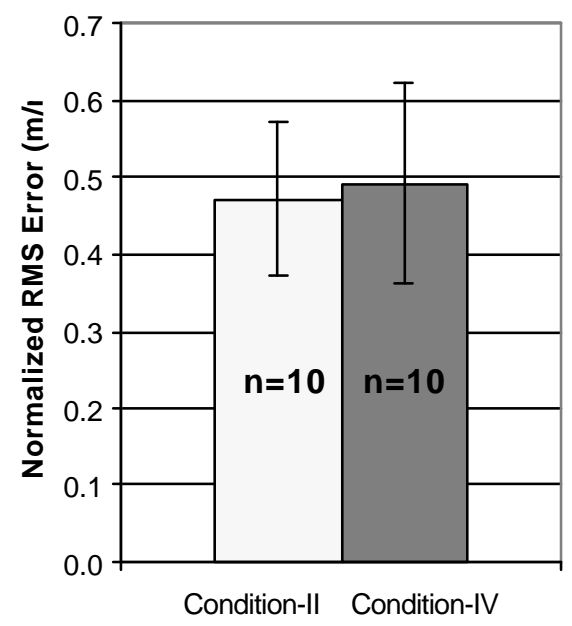

Figure 6. Average of RMS Error for Haptic Conditions A and D (3 Second Time Window) with standard errors of means shown.

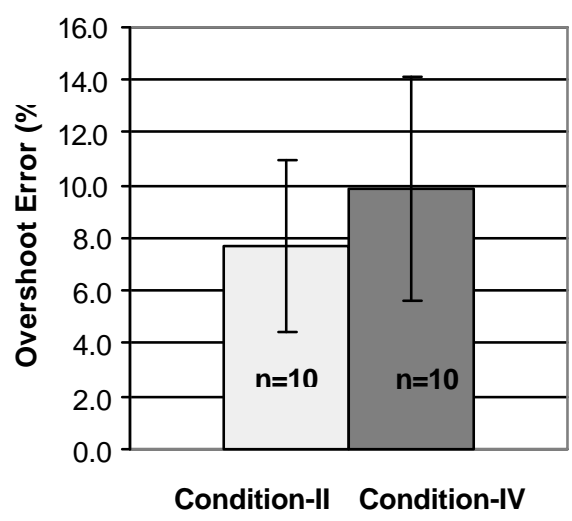

Figure 7. Average Percent First Overshoot Error Comparison of Conditions $A$ and D with standard errors of means shown.

The overshoot data is presented in percentage of the target position, and the norms of error are presented as fractions of unity. In Figures 6 and 7, there are some indications that Condition-II produced less error compared with Condition-IV for overshoot and RMS error. However, in both metrics, the standard deviation of the results are large, and a Student's ttest does not reveal a significant difference under the assumption of equal variance between subjects groups.

To examine the effects of ordering, we present the results for first overshoot error and RMS error for each subject from the first to second session. In Figure 8 and Figure 9, the sequence of presentation of haptic condition is shown, read left to right, and the respective error results are shown for each subject group.

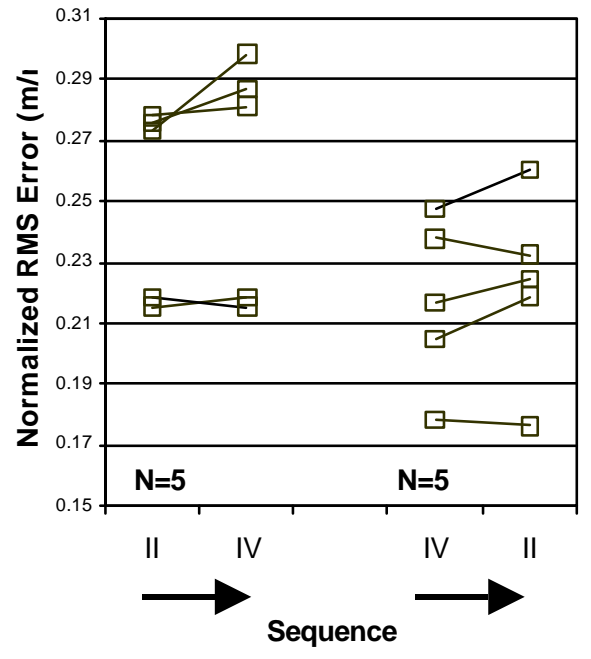

Figure 8. Comparison between Ordering of Haptic Conditions on RMS error (3-Second Window) 


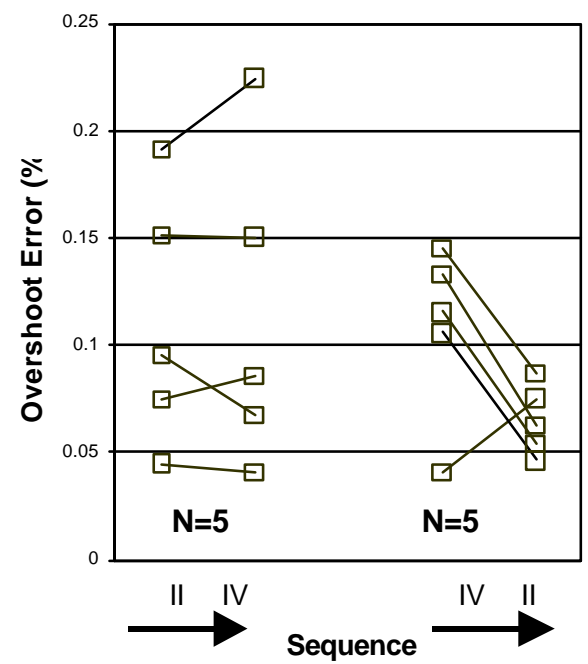

Figure 9. Comparison between Ordering of Haptic Conditions on Overshoot Error

This data represents the performance change in the initial rise period of the response; the same trends were found to continue throughout the settling of the response. Consistent with the conclusion that the larger ball poses a greater challenge, Figures 8 and 9 show general trends of increased RMS error for the II-IV-group, and decreased overshoot error for the IV-II group. Paired t-tests reveals significant differences ( $\mathrm{t}=2.07, \mathrm{df}=6, \mathrm{p}=.042$, one-tailed) for group IV-II in overshoot, but less certain differences for group II-IV in RMS error $(\mathrm{t}=2.05, \mathrm{df}=4, \mathrm{p}=.054$, one-tailed). No strong trends can be observed for the RMS error for the IV-II group, nor for the overshoot error for the II-IV group.

\section{Discussion}

Our analysis first compares the merits of the two haptic conditions. The results of overshoot error show that condition-II did not suffer from the lack of haptic feedback as might be expected. Conditions II and IV produced equivocal results in RMS error suggesting that the presence of more information from force feedback in condition-IV did not in general compensate for the difficulty of the more complex task. On the other hand, as mentioned in Section-1, we may interpret the poor results of the larger mass as being due to a more basic cause, the increased workload of interacting with larger forces. Thus, in terms of utility for closed loop control, higher haptic feedback cannot be assigned merit from these results.

In examining the trends due to ordering effects, we would expect that if there is a positive training effect from the first session, the human might be able to transfer skill from the first session and maintain performance while transitioning to the second. In addition, humans would acquire knowledge about the system and achieve better control through the general experience. It appears, however, that only in the case of group IV-II (transitioning from large to small ball) was there an indication of clear improvement in overshoot error. We can conclude the following: either condition-II was much easier than IV-- a supposition not supported by the other transitions shown in Figures 8 and 9 , or there was a positive training effect of having the large ball precede the small ball for overshoot error. We do not, however, observe a similar trend in RMS error, which suggests that the human's ability to correct error during the control of the ball was not aided by haptic feedback of the large ball.

As discussed in Section 2.1, the choice of haptic condition places a weighting on different aspects of the dynamic behavior of the system. The ordering results for overshoot may indicate that the effect of an initial training period with a large ball may better train subjects for a small ball because of the higher difficulty or the increased intensity of haptic information. The greater workload of interaction with higher forces may even constitute a greater challenge that further prepares subjects in group IV-II to apply high skill to a subsequent easier task. Thus, in terms of overshoot error the utility of practice with the large ball may be in providing system information to the human that is then applicable to the small ball. These results indicate that the prior practice with more difficult haptic conditions could have lasting training effects.

In section 2, our assumption of the human as a flow source that makes use of haptic information must be underscored with the notion that the haptic information arises coupled with power transfer. The merits of more information from larger force feedback may be compromised with greater physical effort requirements. The cost and benefits of more complex dynamics is a factor that may also contribute to the training effect. The results of our current experiment design suggest the need to further isolate the task conditions to determine how the individual factors affect human performance and learning. Referring to Table-1, we may design additional experiments to isolate variation in magnitude and complexity. For example, for a high complexity of interaction, we might test high and low magnitudes of force feedback. 


\section{Conclusions}

For humans, although the primary mode of sensing in common tasks is visual, the haptic mode may be especially important in the control of dynamic tasks. In this paper, we have shown for a simple targeting task using our ball and beam apparatus, human performance is affected by such factors as the magnitude of force feedback and the complexity of the system dynamics. We showed modest evidence that prior exposure to more complex and high feedback conditions improves performance on the simple tasks when performed subsequently.

In future work, we propose to synthesize and haptically render virtual objects whose dynamical behaviors are specifically designed to function as training and rehabilitation therapies. To direct their design, we plan to perform further human subject studies such as the work reported herein and develop an underlying theory of human motor control of dynamical systems. These studies will incorporate learning of novel dynamical tasks with skill acquisition and retention tested through probe-like perturbations to the coupled dynamics.

We aim to present motor tasks in a virtual environment whose dynamics are sufficiently similar to tasks in the physical environment to assure skill transfer, yet might serve as superior skill training and rehabilitation therapies because we can assign a graded difficulty level that can be tailored to the individual's abilities. If we can discover the various roles of force feedback in manipulation, we could differentially promote its learning-supportive roles to create novel robotic training paradigms. We are pursuing various types of assistance, including virtual fixtures (constraints that prevent incorrect motions) virtual agents (coaches that can guide movement, demonstrate strategies, or simply provide additional feedback) and alterations to the apparent laws of physics (changing time constants, for example.

Our current study highlights the complex role of haptic feedback in human motor control performance and motivates a more critical treatment of the various positive and negative effects of force feedback. Through this study, we hope to achieve a formalized understanding of the role of haptic feedback in human-machine interactions that will allow us to design virtual environments for optimal human training.

\section{Acknowledgements}

The authors thank the anonymous reviewers for their insightful comments and the Department of Mechanical Engineering at the University of Michigan for the seed funding in Information Technology that supported this work.

\section{References}

[1] Brooks, Jr., F.P., Ouh-Young, M., Batter, J.J., Kilpatrick, P.J., "PROJECT GROPE: Haptic Displays for Scientific Visualization”, Computer Graphics, 24(4), 177-185, 1990.

[2] Hasser, Christopher J., "User Performance in GUI Pointing Task with a Low Cost Force Feedback Pointer Mouse", Proceedings of the ASME Dynamic Systems and Control Division, DSC-Vol 64, pp. 151-155, ASME 1998.

[3] F. A. Mussa-Ivaldi, J. L. Patton, "Robots can teach people how to move arm", Proceedings of the 2000 IEEE International Conference on Robotics and Automation, pp. 300-305, San Francisco, Ca, April 2000.

[4] Schaal S., Sternard D., Atkeson C. G., "Onehanded Juggling: A dynamical Approach to rhythmic Movement Task", Journal of Motor Behavior, 1996 28,.2, pp. 165-183.

[6] Yamashita Tadashi, Tomoharu Nakamura, Masaaki Ohtuska, Takao Taniguchi, “Observation and Analysis of Heuristic Learning Process in Manual Control of a Crane”, Digital Systems for Industrial Automation, Vol. 2-1, 1983, pp. 31-47.

[7]. D.W. Repperger, C.A. Phillips, and T.L. Chelette, 1995, "A Study on Spatially Induced "Virtual Force" with an Information Theoretic Investigation of Human Performance", IEEE Transactions on Systems, Man, and Cybernetics, 25(10):1392-1404.

[8] Childress, D.S., "Issues Regarding Feedback in Limb Prostheses," Proceedings of the 9th Annual Conference on Rehabilitation Engineering, p. 21, 1986. 